\title{
BRAIN POTENTIAL CHANGES IN MAN INDUCED BY METRAZOL
}

\author{
BY \\ MORTON A. RUBIN AND CONRAD WALL
}

The Memorial Foundation for Neuro-Endocrine Research and the Research Service, Worcester State Hospital, Worcester, Massachusetts.

(RECEIVED 27TH OCtOBER, 1938)

Metrazol therapy in schizophrenia was started at the Worcester State Hospital in the summer of 1937. A study of the influence of metrazol on the electroencephalogram was begun at the same time in the hope of throwing some light on the mechanisms underlying this type of therapy.

Twenty-eight injections of 10-per cent. metrazol (cardiazol or pentamethylenetetrazol) were given to 11 schizophrenic patients. Brain potentials were recorded, starting about 5 minutes before the injection and continuing in some instances for an hour or so.

The recording apparatus was the same as previously described (Rubin, 1938), consisting of two completely independent ink-writing undulators and suitable amplifiers. The electrodes were flattened pellets of lead solder approximately $2 \mathrm{~mm}$. in diameter. The grid leads were fixed on the scalp along the midline over the desired cortical regions ; a reference (earthed) lead was placed behind the ears on the mastoid processes. This technique has, among other things, the advantage of grounding the patient. The administrator of the metrazol was also grounded, so that artifacts resulting from injection of the metrazol and restraining the patient were minimized to a great extent.

\section{Results}

Characteristic alterations of the electro-encephalogram were observed when metrazol evoked an emotional response or a seizure. When neither of these reactions occurred there were no changes in the electrical activity of any of the major regions of the cerebral cortex. The cortical response to metrazol may be divided into three types, according to the most obvious clinical reaction of the patient.

Mild emotional response.-The alpha (10 per second) rhythm was slowed to about 6 per second, when only a mild emotional reaction, usually appearing as a momentary look of apprehension, was induced by metrazol. This occurred 
once in each of four patients. The 6 per second rhythm usually lasted for a minute or so and was followed by a period of rather irregular activity for about 5 minutes, when the pre-metrazol electro-encephalogram was completely restored. These changes are similar to those described by Cook and Walter (1938) for a case in which no seizure occurred.

Severe emotional response.-Metrazol failed to evoke a seizure in one of our patients (A. C.) even though the dosage was raised to 13 c.c. He did show a severe emotional reaction to the metrazol, which was accompanied by deliriform behaviour. Marked flushing of the face and an increase in respiratory rate occurred; he was apprehensive and apparently confused, continually crying, "Please don't give me any more." There was no increase in muscular

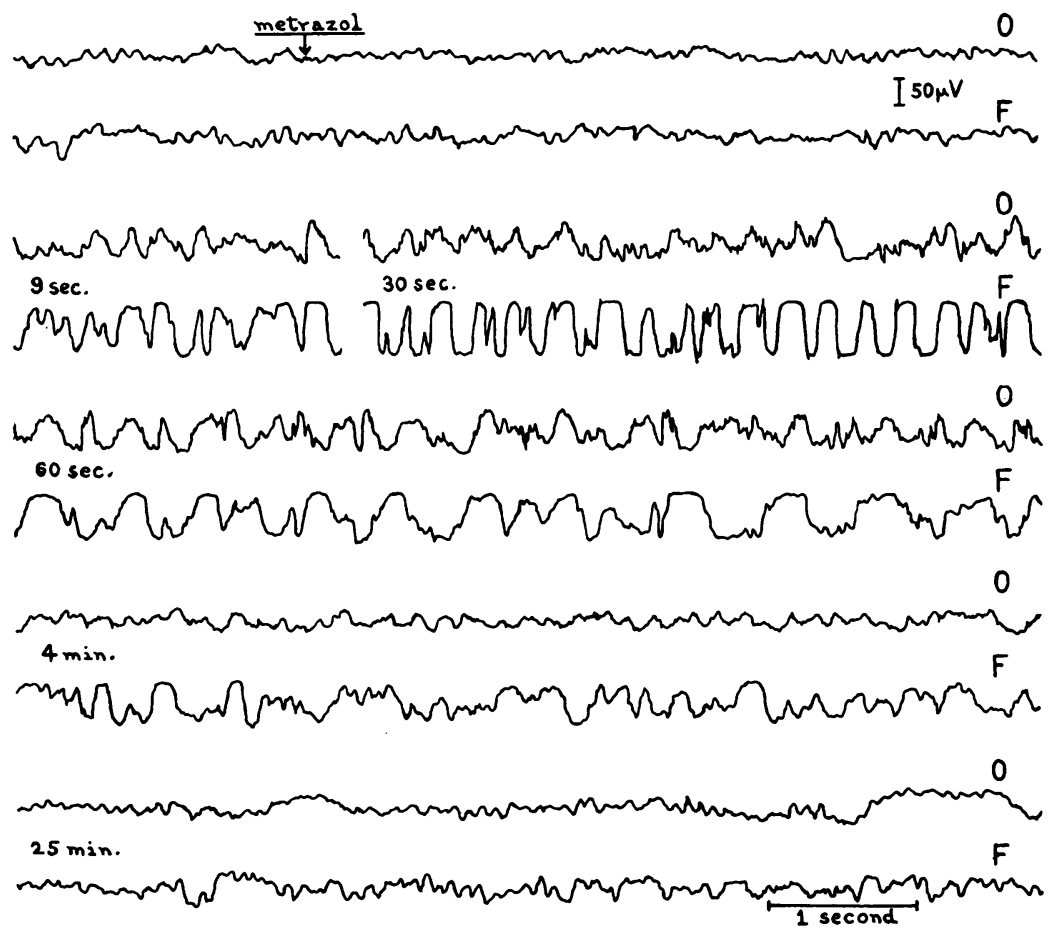

Fig. 1.-Electro-encephalograms from the occipital $(\mathrm{O})$ and frontal $(\mathrm{F})$ regions of patient A. C. during a severe emotional response evoked by 12.5 c.c. of metrazol. Characteristic strips of record illustrate the sudden onset and gradual disappearance of slow rhythms, especially in the frontal lobes. Amplification kept constant throughout.

activity of the type associated with a seizure, yet the tracing from a frontal electrode was strikingly similar to the potential changes during the second myoclonic stage of a seizure. Fig. 1 illustrates the alterations which took place in the occipital and frontal lobes during such an emotional episode. The occipital record became more irregular, exhibiting a frequency of about 6 per second, which finally disappeared by 25 minutes. The frontal lobes showed 
6 per second waves of increased voltage 9 seconds after the metrazol injection ; by 30 seconds a spike-wave pattern with a frequency of 3 to 4 per second appeared, and at 1 minute the frequency was 2 to 3 per second. The record maintained an irregular, mixed pattern, until at 25 minutes it reverted almost completely to the pre-metrazol level. Similar reactions were observed one or more times in two other patients.

As a rule the electrical activity of the frontal lobes only was appreciably altered during a severe emotional episode. Other cortical areas were affected at most to the extent that the occipital record in Fig. 1 shows. The time for complete re-establishment of the pre-metrazol electro-encephalogram was directly proportional to the severity of the emotional response; the more severe the response, the longer the recovery time.

Seizure.-In Fig. 2 are shown characteristic phases of the electro-encephalogram, and their occurrence in time, associated with a metrazol seizure. There
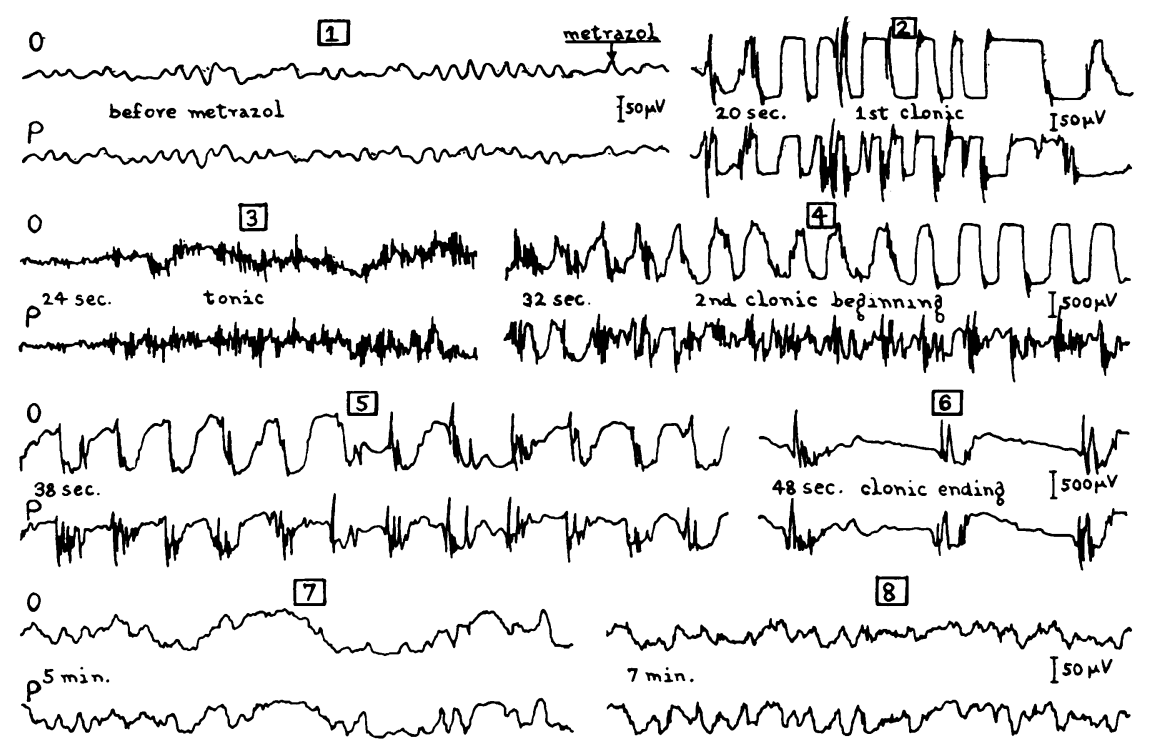

0

9

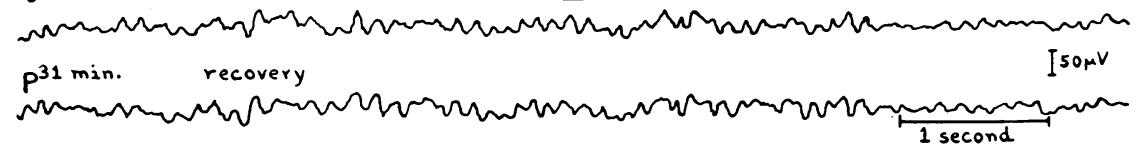

Fig. 2.-Tracings from the occipital (O) and parietal $(\mathrm{P})$ regions of patient W. P., showing the characteristic changes in the electro-encephalogram during a metrazol seizure and recovery from it. See text for description.

are three main stages of muscular activity during typical metrazol convulsions in man, according to evidence from high-speed motion pictures and electromyograms (Strauss and Landis, 1938). The electro-encephalogram also shows 
three phases which are closely associated with the stages of muscular activity. Large (250 microvolts) waves appear 6 to 20 seconds after injection of metrazol, marking the first myoclonic stage, and continue for 3 to 10 seconds. During the tonic stage, which occurs 15 to 30 seconds after injection and lasts for 10 to 30 seconds, the electro-encephalogram is characterized by high-voltage spikes which have the frequency of muscle potentials. The second clonic phase occurs 30 to 50 seconds after the metrazol injection, lasts 25 to 40 seconds, and is associated with cortical potentials at 3 to 4 per second and 1,000 microvolts in amplitude ; they become slower, decrease in voltage, and appear with a spike component toward the end of the seizure. The entire seizure usually took $\frac{1}{2}$ to $1 \frac{1}{2}$ minutes from beginning to end. The time for the onset and duration of the three seizure phases was relatively constant for a given individual from seizure to seizure.

Immediately on cessation of the convulsions, at which time the patient may exhibit severe emotional symptoms, the amplitude of the potential changes dropped markedly and the pattern was one of even slower, but more irregular, activity for the next 5 to 10 minutes. The final phase, lasting until the patient was fully recovered, consisted of an increase in frequency and a dropping out of the irregular, slow waves. Recovery was usually complete in 30 to 80 minutes. This last phase exhibits two of the properties of the electro-encephalogram during sleep (Loomis, Harvey, and Hobart, 1937) : (1) the predominant activity shifted back and forth from an almost completely recovered level (absence of slow waves and a fairly well established alpha rhythm) to one resembling the stage immediately following the seizure ; (2) as early as 10 to 15 minutes before the patient opened his eyes and spoke spontaneously, asking him to speak or to move one of his fingers was answered by speech or by movement of the designated finger ; the electro-encephalogram reverted to the premetrazol level while the patient was responding to the request. Immediately after this, the "drowsy" potential pattern typical of this phase reappeared. These two changes correspond to the shifts in the level of the cortical activity during sleep which occur spontaneously and on arousing the attention of a sleeping subject.

In marked contrast with our findings, Cook and Walter (1938) observed no waves of any sort immediately following the cessation of the convulsions. We believe that this difference is due to the fact that they used bipolar recording. Kornmüller and Schaeder (1938) and Rubin (1938) have discussed the properties of bipolar and monopolar recording in detail, so that it is not necessary to belabour the matter here. Suffice it to point out that bipolar leads placed over regions exhibiting very similar electrical activity will record no appreciable difference in potential between these regions. Monopolar records from the occipital and parietal regions (Fig. 2, strip 7), for example, typical of the cortical activity following the end of a seizure, are strikingly similar.

The "focus" of slow, high-voltage potential changes associated with a metrazol seizure varies considerably, even in the same individual. It may occur in either the occipital, motor, or frontal region. We have never observed it in the parietal area. For example, occipital and vertex records taken on one 


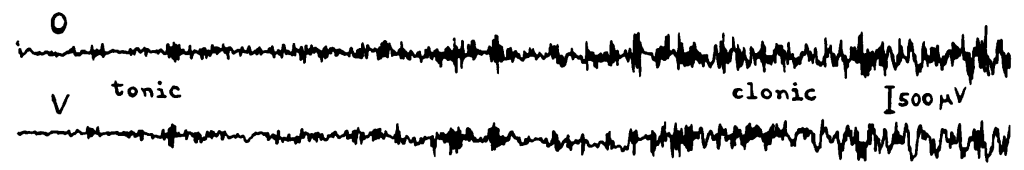

Mintw

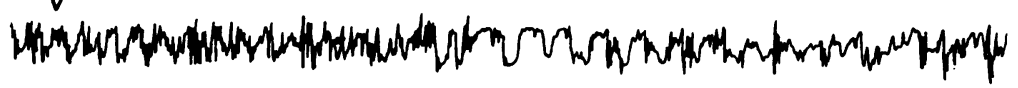
0
$V$

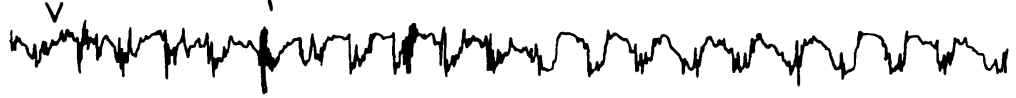

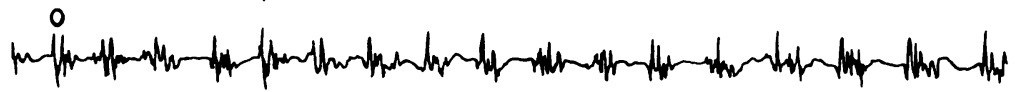

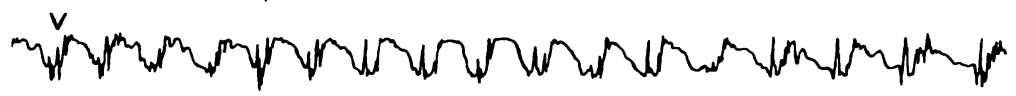

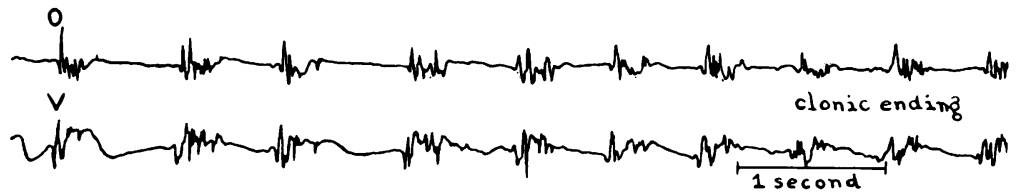
Fig. 3.-Continuous records from the occiput $(\mathrm{O})$ and vertex $(\mathrm{V})$ of patient $\mathrm{W}$. P. during a
metrazol seizure. Amplification constant throughout.

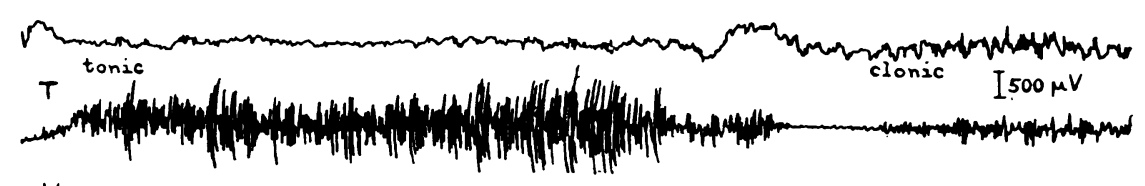

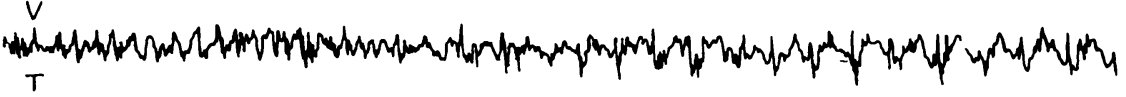

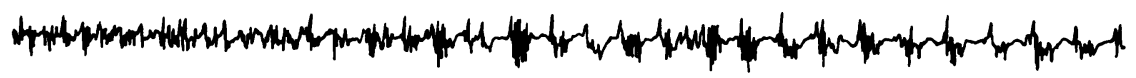

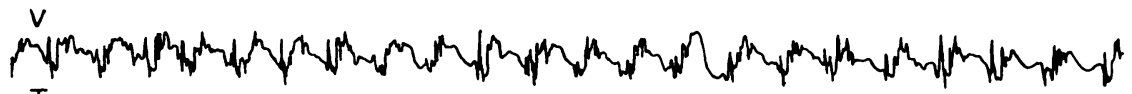

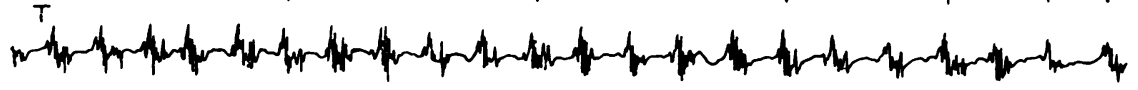

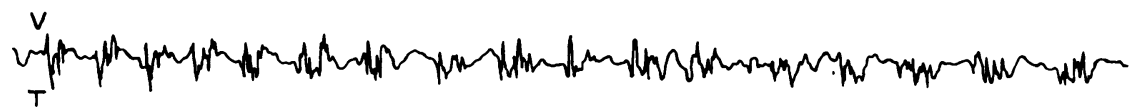

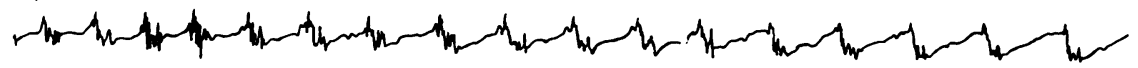

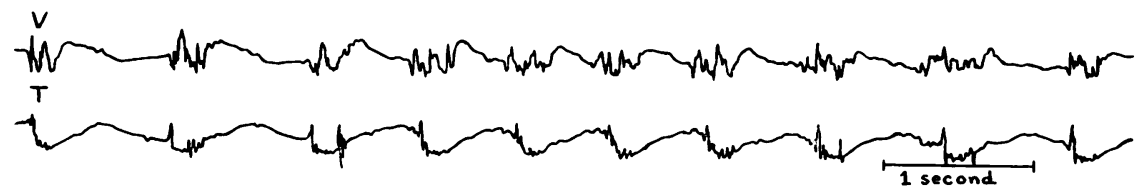

Fig. 4.-Comparison of continuous records of potential changes from the vertex (V) and the triceps muscle $(\mathrm{T})$ during a metrazol seizure. Amplification constant throughout. 
day from patient W. P. (Fig. 3) showed no appreciable slow activity under the occipital lead, although it was very marked in the motor region. Compare the occipital record in Fig. 3 with that in Fig. 2, obtained from the same patient during another seizure. On the basis of frequency of occurrence in our observations, the motor region was the most common "focus" of slow activity. Our data, however, are not sufficiently numerous to designate any one area as a "focus." Cook and Walter (1938) believe that the primary "focus" is in the frontal region.

A metrazol seizure is so violent that a reasonable doubt might arise as to whether or not the brain potential changes associated with it are really nervous in origin. Simultaneous records from the motor area and the triceps muscle of the left arm (Fig. 4), however, demonstrate that these potentials are not due to the spread of muscle potentials, for the following reasons : (1) If the seizure electro-encephalogram were merely the result of muscle potential spread, we should expect the high-voltage discharge in the triceps lead during the tonic contractions to appear in the vertex record. This is not the case. (2) The slow brain potentials are, in the main, of considerably higher voltage and have different wave-form than the accompanying slow muscle potentials. (3) In the last part of the clonic stage, the spike potentials from the motor region are of greater amplitude than the corresponding muscle potentials. (4) Although the speed with which the recording paper moved was not great enough to decide the point definitely, it is our impression that the cortical spike-wave formation precedes the similar muscle potential pattern.

\section{Discussion}

Metrazol produces a general anoxia (Himwich et al., 1937). The electroencephalogram in anoxia is similar to that found in a "depressed" cerebral cortex, namely one of high amplitude and low frequency (Gibbs, Davis, and Lennox, 1935). Rubin and Freeman (1938) have found that sub-convulsive doses of sodium cyanide produce 3 to 6 per second waves when the cortex is functionally "depressed" (as in narcolepsy and in catatonic stupor). They concluded that cyanide acts directly on the cortical neurons, enhancing the activity of the neurons. The same may be true in the case of the slow waves induced by metrazol.

According to Maurer et al. (1938), the $p \mathrm{H}$ value of the blood decreases markedly during metrazol convulsions, starting with the tonic phase, reaching a minimum at the end of the clonic phase, and increasing again to its premetrazol value some time after 20 minutes. The decrease in $p \mathrm{H}$ is probably due to the increase in lactic acid produced by the violent muscular activity during the seizure. The maximum lactic acid value was found 10 to 20 minutes after injection of the metrazol, and it returned to normal in 45 to 60 minutes. The changes in blood chemistry resulting from metrazol probably contribute only secondarily in so far as the electro-encephalogram during the seizure is con- 
cerned. Dusser de Barenne, McCulloch, and Nims (1937) have shown that a decrease in $p \mathrm{H}$ of the cortex results in a decrease of frequency and amplitude of cortical potentials. This is probably reflected in the drop in amplitude and the slowing of frequency toward the end of the second myoclonic phase of the seizure and the period immediately following (Fig. 2), which corresponds in time to the minimum $p \mathrm{H}$ value of the blood. It is interesting that the electroencephalogram reverts to the pre-metrazol level at about the same time that the blood constituents return to their normal values.

When metrazol fails to produce convulsions no change is observed in blood $p \mathrm{H}$ (Maurer et al., 1938), which may account for the more rapid recovery of the electro-encephalogram after an emotional response than after a seizure. Metrazol does cause a pronounced sympathetic discharge, which is accentuated if anoxia exists (Gellhorn, 1938). "Emotional" stimulation has been shown to increase the amount of slow brain potential changes (Hoagland, Cameron, and Rubin, 1938).

The mechanism of the alterations in the electrical activity of the cortex during an emotional reaction induced by metrazol is probably somewhat different from that involved when a seizure occurs. A marked sympathetic discharge evoked by sub-convulsive doses of metrazol, especially if doses of metrazol also produce anoxia, may set off the slow rhythms characteristic of an emotional response. This seems reasonable if we consider that the slow potential changes during an emotional episode are of much lower voltage than those during a seizure and that they arise primarily in the frontal lobes, which are well supplied with thalamo-cortical fibres. Since the blood chemistry is not altered in the absence of convulsions, the electro-encephalogram reverts to its pre-metrazol pattern more rapidly than after a seizure, the time for full recovery depending primarily on how severe the emotional response to the metrazol was.

\section{Summary}

Alterations of the electro-encephalogram and electromyogram in 11 schizophrenic patients before, during, and after the emotional response or seizure induced by intravenous injection of metrazol are described.

Changes in blood chemistry produced by metrazol are related to the alterations observed in the electro-encephalogram.

\section{REFERENCES}

Cook, L. C., and Walter, W. G. (1938). J. Neurol. Psychiat., 1, 180.

Dusser de Barenne, J. G., McCulloch, W. S., and Nims, L. F. (1937). J. cell. comp. Physiol., $10,277$.

Gellhorn, E. (1938). Arch. Neurol. Psychiat., 40, 125.

Gibbs, F. A., Davis, H., and Lennox, W. G. (1935). Ibid., 34, 1,133.

Himwich, H. E., Bowman, K. M., Fazekas, J. F., and Orenstein, L. L. (1937). Proc. Soc. exp. Biol. N.Y., 37, 359. 
Hoagland, H., Cameron, D. E., and Rubin, M. A. (1938). J. gen. Psychol., 19, 227.

Kornmüller, A. E., and Schaeder, J. A. (1938). J. Neurophysiol., 1, 287.

Loomis, A. L., Harvey, E. N., and Hobart, G. A. (1937). J. exp. Psychol., 21, 127.

Maurer, S., Wiles, H. O., Marberg, C. M., Skorodin, B., and Fisher, M. L. (1938). Amer. J. Psychiat., 94, 1,355.

Rubin, M. A. (1938). J. Neurophysiol., 1, 313.

Rubin, M. A., and Freeman, H. (1938). J. Neurophysiol., 1, 527.

Strauss, H., and Landis, C. (1938). Proc. Soc. exp. Biol. N.Y., 38, 369. 